Geometry $\&$ Topology

Volume 9 (2005) 2129-2158

Published: 4 November 2005

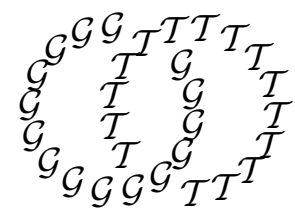

\title{
New topologically slice knots
}

\author{
STEFAN FRIEDL \\ Peter Teichner \\ Department of Mathematics, Rice University \\ Houston, TX 77005, USA \\ and \\ Department of Mathematics, University of California \\ Berkeley, CA 94720, USA \\ Email: friedl@rice.edu and teichner@math.berkeley.edu
}

\begin{abstract}
In the early 1980's Mike Freedman showed that all knots with trivial Alexander polynomial are topologically slice (with fundamental group $\mathbb{Z}$ ). This paper contains the first new examples of topologically slice knots. In fact, we give a sufficient homological condition under which a knot is slice with fundamental group $\mathbb{Z} \ltimes \mathbb{Z}[1 / 2]$. These two fundamental groups are known to be the only solvable ribbon groups. Our homological condition implies that the Alexander polynomial equals $(t-2)\left(t^{-1}-2\right)$ but also contains information about the metabelian cover of the knot complement (since there are many non-slice knots with this Alexander polynomial).
\end{abstract}

\section{AMS Classification numbers Primary: 57M25}

Secondary: $57 \mathrm{M} 27,57 \mathrm{~N} 70$

Keywords: Slice knots, surgery, Blanchfield pairing

Proposed: Robion Kirby

Seconded: Cameron Gordon, Wolfgang Lueck
Received: 12 May 2005

Accepted: 10 October 2005 


\section{Introduction}

A knot is an embedding $S^{1} \hookrightarrow S^{3}$. We work in the topological category and assume that every embedding is locally flat. Note that a smooth embedding is locally flat and that we prefer to add the adjective "smooth" to emphasize this stronger condition on the embedding, except in our title. Two knots are called concordant if there exists an embedding $S^{1} \times[0,1] \hookrightarrow S^{3} \times[0,1]$ which restricts to the given knots at both ends. The concordance classes form an abelian group under connected sum, the knot concordance group $\mathcal{C}$. A knot is called slice if it is concordant to the unknot or, equivalently, if it bounds an embedding of disks $D^{2} \hookrightarrow D^{4}$. Predating the 4-dimensional revolution in the early 80's, Casson and Gordon showed that the epimorphism from $\mathcal{C}$ onto its high dimension analogue has a nontrivial kernel [2]. There has been much recent progress in understanding how complicated $\mathcal{C}$ really is. In [5], [6] an infinite sequence of new invariants was found using non-commutative Blanchfield forms and their von Neumann signatures. On the other hand, many knots are known to be slice, for example the knots in Figure 1.1. where the band can be tied into an arbitrary knot $C$. In fact, there is a large class of slice knots given as the

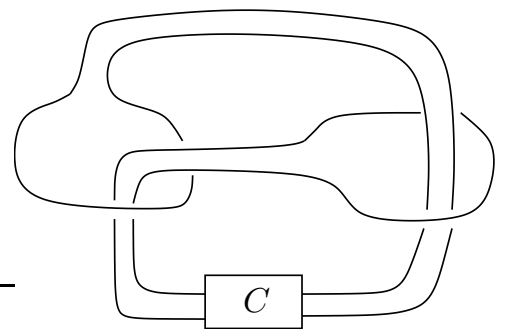

Figure 1.1: A family of ribbon knots

boundary of ribbons in $S^{3}$. These knots are called ribbon knots, where a ribbon is a smooth immersion $D^{2} \rightarrow S^{3}$ such that all singularities are of the type as in Figure 1.2. They consist of arcs of self-intersection that lie completely in the interior of one of the two sheets involved. Such singularities can be resolved in $D^{4}$ by pushing an open disk around each singular arc slightly away from $\partial D^{4}$. Thus a ribbon leads to a smooth slice disk in $D^{4}$, the so called ribbon disk. It is a fascinating open problem whether every smoothly slice knot is ribbon. One distinctive feature of a ribbon knot is that the inclusion map induces an epimorphism of the knot group onto the ribbon group, the fundamental group of the complement in $D^{4}$ of the ribbon disk. There is a simple criterion for a given group to be ribbon in terms of certain presentations, see Theorem 2.1. For 
general slice complements the inclusion map does not induce an epimorphism, and slice disks with the ontoness property are called homotopically ribbon, or $h-$ ribbon for short.

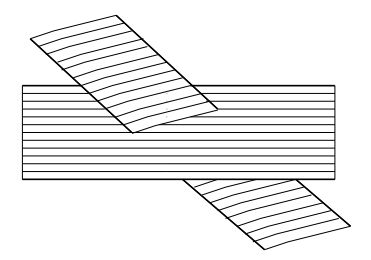

Figure 1.2: Local singularity of a ribbon

In the topological category, Freedman proved that any knot with trivial Alexander polynomial is slice [10], see also [13] for a more direct construction. Using gauge theory, Gompf showed that some of these knots are not smoothly slice 14. The easiest such knot is probably the Whitehead double of the trefoil knot, already exhibiting the subtle difference between smooth and topological 4-manifolds. In an amazing turn of events, Rasmussen very recently gave the first purely combinatorial proof for the fact that this knot is not smoothly slice. He constructed a concordance invariant from Khovanov homology [28] with beautiful properties. In particular, the arguments of [22] for showing that (iterated) Whitehead doubles of the trefoil are not smoothly slice can be adapted to this setting from the concordance invariant of Ozsváth and Szabó [23]. Livingston uses the following argument, going back to at least Rudolph: One can exhibit some Whitehead doubles as separating curves on minimal Seifert surfaces of certain torus knots. Since the Rasmussen and Ozsváth-Szabó invariants detect the minimal genus of torus knots, it follows that such separating curves cannot be smoothly slice.

In this note we provide the first new class of slice knots since Freedman's construction, using his theorem [9] that solvable groups are good (for topological surgery). Our main result is the following theorem. Let

$$
S R:=\left\langle a, c \mid a c a^{-1}=c^{2}\right\rangle \cong \mathbb{Z} \ltimes \mathbb{Z}[1 / 2] .
$$

Here the generator $a$ of $\mathbb{Z}$ acts on the normal subgroup $\mathbb{Z}[1 / 2]$ via multiplication by 2 . It is known, cf Lemma 2.2, that $S R$ and $\mathbb{Z}$ are the only solvable ribbon groups, hence the name. In geometric group theory, this group is also known as the Baumslag-Solitar group B(1,2).

Theorem 1.3 Let $K$ be a knot and denote by $M_{K}$ the 0 -surgery on $K$. If there is an epimorphism $\pi_{1}\left(M_{K}\right) \rightarrow G, G=S R$ or $\mathbb{Z}$, such that

$$
\operatorname{Ext}_{\mathbb{Z}[G]}^{1}\left(H_{1}\left(M_{K} ; \mathbb{Z}[G]\right), \mathbb{Z}[G]\right)=0
$$


then $K$ is (topologically) slice. In fact, $K$ is h-ribbon with group $G$ if and only if this Ext-condition holds for some epimorphism $\pi_{1}\left(M_{K}\right) \rightarrow G$.

The case $G=\mathbb{Z}$ is actually just a reformulation of Freedman's theorem because the condition (Ext) is then equivalent to $\Delta_{K}(t)=1$. For $G=S R$, we shall show in Corollary 3.4 that this condition implies

$$
\Delta_{K}(t)=(t-2)\left(t^{-1}-2\right) .
$$

There are well known knots with this Alexander polynomial that are not slice (cf Section 17) so the h-ribbon question with group $S R$ is more subtle than for $\mathbb{Z}$. Our result complements work of Tim Cochran and Taehee Kim [7. They show that if the degree of the Alexander polynomial is greater than two, than the homology of solvable covers can not determine whether a given knot is slice or not.

Remark We will show in Lemma 5.1 that the somewhat awkward (Ext) condition can be replaced by the condition that a non-commutative Blanchfield pairing

$$
B \ell: H_{1}\left(M_{K} ; \mathbb{Z}[G]\right) \times H_{1}\left(M_{K} ; \mathbb{Z}[G]\right) \longrightarrow Q(G) / \mathbb{Z}[G]
$$

vanishes, where $Q(G)$ denotes the Ore localization of $\mathbb{Z}[G]$.

It is surprisingly easy to construct many knots that satisfy all conditions of our Theorem 1.3. For example, all knots in Figure 1.1 do. The easiest one is the knot $6{ }_{1}$, which is isotopic to the case where the band $C$ is the unknot. However, all knots in Figure 1.1 are obviously ribbon and hence we need to work harder to get new $\mathrm{h}$-ribbon knots. One trick is the following satellite construction that can be considered as an analogue of Whitehead doubling (a nice way to obtain knots with trivial Alexander polynomial). We give a much more general result in Section [6.

Theorem 1.4 Start with the knot $6_{1}$, drawn in a solid torus as on the right hand side of Figure 1.5. Tie an arbitrary knot into that solid torus to obtain a satellite $K$ of $61_{1}$. Then $K$ satisfies the assumptions of Theorem [1.3 and is therefore h-ribbon.

On the left hand side of Figure 1.5 we picked an axis $A$ which is unknotted in $S^{3}$ (so that the complement of $A$ will be a solid torus) and which punctures all ribbon disks that we could see. Then we redrew the picture in a way that $A$ becomes the meridian to the visible solid torus. We conjecture that Theorem 1.4 

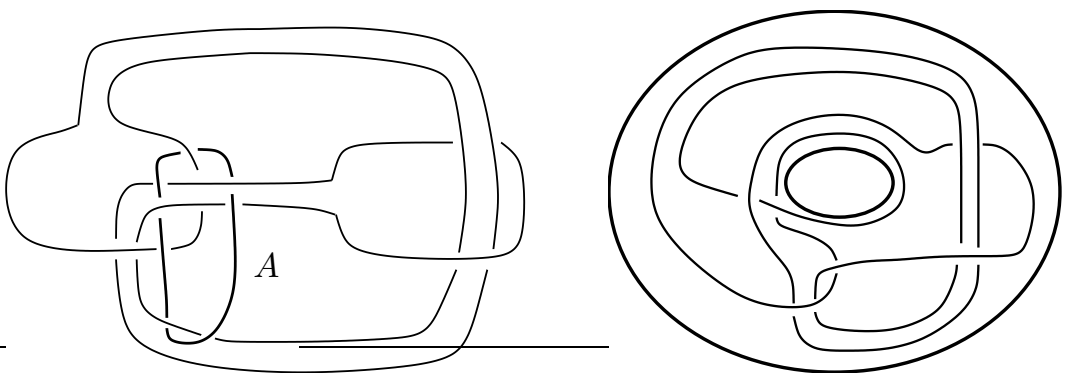

Figure 1.5: The knot $6_{1}$ in the solid torus $S^{3} \backslash A$

gives new examples of knots which are $\mathrm{h}$-ribbon but not smoothly slice. The simplest candidate is the satellite knot of the trefoil knot. It has a knot diagram with 93 crossings and we are unable to compute Rasmussen's invariant [28], the complexity of which is exponential in the number of crossings. For the general ribbon case we propose the following generalization of Theorem 1.3

Conjecture 1.6 Let $G$ be a ribbon group for which topological surgery works. A knot $K$ is h-ribbon with group $G$ if and only if there exists an epimorphism $\varphi: \pi_{1}\left(M_{K}\right) \rightarrow G$ such that the condition (Ext) from Theorem [1.3 holds.

Note that the converse of this conjecture is not completely straight forward either. We use a Blanchfield form to prove the converse for the groups $G=S R$ and $\mathbb{Z}$.

Question 1.7 Are the fundamental groups of complements of $h$-ribbons ribbon groups?

The phrase "surgery works for $G$ " means here that the (reduced) surgery sequence

$$
\mathcal{S}_{T O P}^{h}(X, M) \longrightarrow \widetilde{\mathcal{N}}_{T O P}(X, M) \longrightarrow \widetilde{L}_{4}^{h}(\mathbb{Z}[G])
$$

is exact for all Poincaré pairs $(X, M)$ with $\pi_{1}(X)=G$, see [10, chapter 11.3] for more information. If $G=S R$ or $\mathbb{Z}$ then this sequence is exact by Freedman's disk embedding theorem (cf [9] and [1] ) for solvable groups. The general case is still open and since most other ribbon groups contain free groups we need a very strong form of this result. It is logically possible that topological surgery works for a given fundamental group but the disk embedding theorem (and hence the s-cobordism theorem) fail for this group.

The "if"-direction of Conjecture [1.6 would follow from the following purely homological conjecture. We say that $\left(M_{K}, \varphi\right)$ satisfies the Poincaré duality 
condition if the induced inclusion $M_{K} \hookrightarrow K(G, 1)$ is a finite 4-dimensional Poincaré pair.

Conjecture 1.8 Let $K$ be a knot with an epimorphism $\pi_{1}\left(M_{K}\right) \rightarrow G$ onto a ribbon group such that the condition (Ext) from Theorem [1.3 holds. Then $\left(M_{K}, \varphi\right)$ satisfies the Poincaré duality condition. Moreover, $\widetilde{L}_{4}^{h}(\mathbb{Z}[G])=0$.

We give supporting evidence for this algebraic conjecture at the end of Section 2 , For $G=\mathbb{Z}$ it is true out of easy reasons and in Lemmas 3.3, 4.1 and Lemma 4.4 we prove that the conjecture is true for $G=S R$. The main reason why this works is the fact that the group ring $\mathbb{Z}[S R]$ is an Ore-domain and hence has an ordinary (skew) quotient field, see Section 4. We use various lemmas and ideas from [5]. The first statement of Theorem 1.3 is then a consequence of the following general result.

Theorem 1.9 Let $G$ be a finitely presented group for which topological surgery works and with $H_{1}(G) \cong \mathbb{Z}, H_{2}(G)=0$ and $\widetilde{L}_{4}^{h}(\mathbb{Z}[G])=0$. A knot $K$ is $h$-ribbon with group $G$ if there is an epimorphism $\varphi: \pi_{1}\left(M_{K}\right) \rightarrow G$ such that $\left(M_{K}, \varphi\right)$ satisfies the Poincaré duality condition. In particular, Conjecture 1.6 follows from Conjecture 1.8.

Theorem 1.9 comes from the fact that given a Poincaré pair, one can attempt to use classical surgery theory to find a 4 -manifold $W$ of type $K(G, 1)$. We show that in the situation above, this approach successfully produces a $\mathrm{h}$-ribbon complement that is a $K(G, 1)$.

The paper is organized as follows. In Section 2 we recall several known facts about ribbon groups. In Section 3 we explain how surgery can be used to find h-ribbons. We accumulate several restrictions on the group $G$, and in Section 4 we show that the group $S R$ satisfies all of them. In Section 5 we will show that for our groups the (Ext)-condition can be replaced by a vanishing condition on a non-commutative Blanchfield pairing. In Section 6 we recall the satellite construction which we use to give examples of topologically slice knots in Section 7.

Acknowledgments We thank Chuck Livingston and the referee for their valuable comments on the first version of this paper. We also wish to thank Jerry Levine and Andrew Ranicki for helpful discussions regarding certain technical aspects in this paper.

The first author was partially supported by RTN Network HPRN-CT-200200287: Algebraic K-Theory, Linear Algebraic Groups and Related Structures. The second author is partially supported by NSF Grant DMS-0453957. 


\section{Ribbon disk complements}

Definition A group $G$ is called ribbon if there exists a ribbon disk $D \hookrightarrow D^{4}$, as explained in the introduction, with $\pi_{1}\left(D^{4} \backslash D\right) \cong G$.

The deficiency of a presentation of a group is the number of generators minus the number of relations. The deficiency $\operatorname{def}(G)$ of a finitely presented group $G$ is the maximum of the deficiencies of all presentations. The following theorem is well-known and uses the fact that any ribbon in 3-space can be obtained as follows: Start with an $s$-component unlink and add $(s-1)$ bands to produce a knot, where the bands can be arbitrarily twisted and linked. In particular, the bands may hit the disks bounding the unlink which introduces our ribbon singularities. This description actually gives an embedded disk $D \hookrightarrow D^{4}$ with a Morse function $D^{4} \rightarrow[0,1]$ which has $s$ minima and $(s-1)$ saddles when restricted to $D$. This implies that the complement $N_{D}:=D^{4} \backslash \nu D, \nu D$ an open tubular neighborhood of $D$, has a handle decomposition with one 0 -handle, $s$ 1-handles and $(s-1) 2$-handles, giving the desired group presentation.

Theorem 2.1 A group $G$ is ribbon if and only if $H_{1}(G)=\mathbb{Z}$ and $G$ has a Wirtinger presentation of deficiency one, ie, $G$ has a presentation of the form

$$
G=\left\langle g_{1}, \ldots, g_{s} \mid r_{1}, \ldots, r_{s-1}\right\rangle,
$$

where $r_{i}=g_{h_{i}} g_{l_{i}}^{\epsilon_{i}} g_{k_{i}}^{-1} g_{l_{i}}^{-\epsilon_{i}}$ for some $h_{i}, k_{i}, l_{i} \in\{1, \ldots, s\}$ and $\epsilon_{i} \in\{-1,1\}$.

Using the above theorem and the Wirtinger presentation, one sees that knot groups are ribbon. As pointed out by John Stallings, a direct geometric way to see the corresponding ribbon disk is as follows. Represent the knot by an arc ending in a plane $P \subset \mathbb{R}^{3}$. A rotation by 180 degrees in $\mathbb{R}^{4}$, with fixed plane $P$, sweeps out a disk in $D^{4}$ which has the same local minima and saddles as the original knotted arc (when projected orthogonally to the fixed plane) and there are no maxima. Applying this construction to a knot $K$, one obtains a ribbon disk with fundamental group $\pi_{1}\left(S^{3} \backslash K\right)$ and boundary $K \#(-K)$. We recover by this argument the reason why the mirror image $-K$ is the inverse of $K$ in the concordance group.

Lemma 2.2 The only solvable ribbon groups are $\mathbb{Z}$ and $S R=\mathbb{Z} \ltimes \mathbb{Z}[1 / 2]$.

Proof Wilson 32 shows that every solvable group of deficiency one is isomorphic to $G_{k}:=\mathbb{Z} \ltimes \mathbb{Z}\left[t, t^{-1}\right] /(t k-1)$ for some $k$. A direct computation shows that $G_{k} /\left[G_{k}, G_{k}\right]=\mathbb{Z}$ if and only if $k=0,2$. These are exactly the two groups in the statement. Below we show that $S R$ is indeed a ribbon group. 
We now show that $S R$ does in fact appear as the fundamental group of a ribbon disk complement. Let $D$ be the ribbon disk given by Figure 1.1. The corresponding ribbon disk complement $N_{D}$ is given by the handle diagram of Figure 2.3, with two (dotted) 1-handles and one 2-handle. This is the case $s=2$ in the discussion above Theorem 2.1] see also [15, p. 213]. Note that with

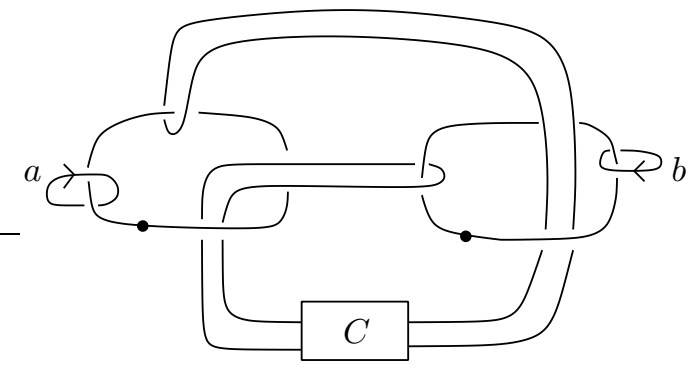

Figure 2.3: Handle decomposition of $N_{D}$, with generators $a, b$ for $\pi_{1}\left(N_{D}\right)$.

$c:=a b^{-1}$ one gets

$$
\pi_{1}\left(D^{4} \backslash D\right)=\left\langle a, b \mid a^{-1} b a^{-1} b a b^{-1}\right\rangle=\left\langle a, c \mid a c a^{-1}=c^{2}\right\rangle=S R .
$$

which is in fact independent of the knot $C$ that was tied into the band in Figure 1.1.

Remark Writing $R(C)$ for the above ribbon knot, then $R(C)$ is a satellite knot with companion $C$ and orbit $R:=R$ (trivial knot). The knot $R$ is the knot $6_{1}$ of the standard knot table and we shall construct many more examples in this manner in Section 6 .

Remark Since any ribbon group $\pi_{1}\left(N_{D}\right)$ has a presentation of deficiency $\operatorname{rank}\left(H_{1}\left(N_{D}\right)\right)=1$ it is in particular an E-group (cf 30. p. 324]), hence $\pi_{1}\left(N_{D}\right)^{(1)}$ is an E-group (cf [30, p. 302]). It follows from a result of Roushon 29. Corollary 4.6] in the case that $\operatorname{rank}_{\mathbb{Z}}\left(\pi_{1}\left(N_{D}\right)^{(1)} / \pi_{1}\left(N_{D}\right)^{(2)}\right) \geq 2$ that the derived series of $\pi_{1}\left(N_{D}\right)$ never stabilizes, ie, $\pi_{1}\left(N_{D}\right)^{(i)} \neq \pi_{1}\left(N_{D}\right)^{(i+1)}$ for all $i \in \mathbb{N}$ (cf also [3]). This means that with few exceptions ribbon groups are neither solvable nor does their derived series stabilize.

\section{The ribbon group conjecture}

Alexander duality implies that ribbon groups satisfy $H_{1}(G) \cong \mathbb{Z}$ and $H_{2}(G)=$ 0 . We say that a group $G$ is aspherical if $K(G, 1)$ is a $2-$ complex. For a ribbon 
group $G=\pi_{1}\left(D^{4} \backslash D\right)$ this is the case if $\pi_{2}\left(D^{4} \backslash D\right)=0$. It is conjectured that ribbon groups are aspherical. Note that this is in turn a special case of the Whitehead conjecture. This conjecture is known to be true for all knot groups since knot complements are aspherical by the sphere theorem. It is also known for all locally indicable ribbon groups (cf [16]), which in particular includes all ribbon groups with $G^{(\alpha)}=\{e\}$ for some ordinal $\alpha$ (cf also 30]). For some more examples cf [17]. In particular, we conjecture that a ribbon group $G$ has the property that

$$
H_{3}(G)=0 \text { and } \operatorname{Ext}_{\mathbb{Z}[G]}^{i}(\mathbb{Z}, \mathbb{Z}[G])=0 \text { for } i>2 .
$$

This conjecture clearly holds for aspherical ribbon groups. Furthermore we conjecture that for a ribbon group $G$ and an epimorphism $\varphi: \pi_{1} M_{K} \rightarrow G$, we always have

$$
\operatorname{Hom}_{\mathbb{Z}[G]}\left(H_{i}\left(M_{K} ; \mathbb{Z}[G]\right), \mathbb{Z}[G]\right)=0 \text { for } i=1,2 .
$$

The relevance of these properties comes from Lemma 3.3 and Theorem 1.9. We will prove all these properties for $G=S R$ in Lemma 4.1

\section{Proof of the Main Theorem 1.3}

Let $K$ be a knot in $S^{3}$ and denote by $M_{K}$ the result of zero framed surgery along $K$. The following is a well known slice criterion, see eg, [5]. For the "only if" direction one takes $W$ to be the complement in $D^{4}$ of a (thickened up) slice disk. For the "if" direction one uses Freedman's solution of the topological Poincaré conjecture in dimension 4 , in order to recognize as $D^{4}$ the 4 -manifold $W$ union a 2 -handle along a meridian for $K$ in $M_{K}$.

Proposition 3.1 A knot $K$ is slice if and only if $M_{K}^{3}$ bounds a 4 -manifold $W^{4}$ with

(1) $\pi_{1}(W)$ is normally generated by the image of a meridian for $K$,

(2) $H_{1}(W) \cong \mathbb{Z}$

(3) $H_{2}(W)=0$

Furthermore, $K$ is $h$-ribbon with group $\pi_{1}(W)$ if and only if $(1)$ is replaced by $(1-\mathrm{h}) \pi_{1}\left(M_{K}\right) \rightarrow \pi_{1}(W)$ is surjective.

Note that the first two conditions can always be satisfied, even with $\pi_{1}(W) \cong \mathbb{Z}$. However, to satisfy condition (3) as well, it is often necessary to make the fundamental group of $W$ more complicated. In fact, if (1)-(3) are satisfied for $\pi_{1}(W) \cong \mathbb{Z}$ then $K$ has vanishing Alexander polynomial. Thus the main problem is to find a candidate for the fundamental group of $W$. 


\subsection{Outline of the construction of $\mathrm{W}$}

To prove Theorem 1.3. we take our candidate for the fundamental group to be $G=S R$ or $\mathbb{Z}$. By assumption we have given an epimorphism $\varphi: \pi_{1} M_{K} \rightarrow G$ and we would like to find a $K(G, 1)$-manifold $W$ with boundary $M_{K}$ such that the inclusion induces $\varphi$. Since any ribbon group satisfies $H_{1}(G)=\mathbb{Z}$ and $H_{2}(G)=0$, such a manifold $W$ would fulfill the conditions (1-h), (2) and (3) above. This means that we are in a classical surgery situation and we can follow the steps taken by many before us. Below, the steps are labelled by the section numbers where they will be worked out.

(3.2) First we seek conditions such that $\left(K(G, 1), M_{K}\right)$ is a Poincaré pair. It turns out that exactly condition (Ext) from Theorem 1.3 arises.

(3.3) Secondly, we check that the Spivak normal bundle of this Poincaré pair has a linear reduction, ie, that there is a degree 1 normal map from a (smooth) manifold pair $\left(N, M_{K}\right) \rightarrow\left(K(G, 1), M_{K}\right)$. In fact, we shall see that there is a unique normal cobordism class of such maps if we require, as we shall, that the signature of $N$ is zero.

(3.4) This normal map has surgery obstruction in the reduced L-group $\widetilde{L}_{4}^{h}(\mathbb{Z}[G])$. Since $\pi_{2} K(G, 1)=0$ this quadratic form is nothing but the intersection form on $\pi_{2} N$. We will verify in Lemma 4.4 that our L-group vanishes and hence the intersection form on $\pi_{2} N$ is (stably) hyperbolic.

(3.5) Finally, since by assumption the surgery sequence $(\mathcal{S})$ for our fundamental groups is exact (in the topological category), one gets a topological manifold $W$ together with a homotopy equivalence $\left(W, M_{K}\right) \rightarrow$ $\left(K(G, 1), M_{K}\right)$. Hence we found our h-ribbon by Proposition 3.1 .

In the following sections, we go through these steps one by one. We will not assume from the beginning that $G=S R$ or $\mathbb{Z}$ but we shall add conditions on $G$ (and $\varphi$ ) as we go, in turn proving Theorem 1.9. It will turn out that some of these conditions are known for all ribbon groups and some are conjectural. For the groups $S R$ and $\mathbb{Z}$ we will prove all conditions in Section 4 .

\subsection{The Poincaré duality condition}

We will use the following conventions. If $\tilde{X} \rightarrow X$ is the universal cover then $C_{*}(\tilde{X})$ has a right $\mathbb{Z}\left[\pi_{1}(X)\right]$-module structure. Given a left $\mathbb{Z}\left[\pi_{1}(X)\right]$-module

$P$ we can consider $H_{*}(X ; P):=H_{*}\left(C_{*}(\tilde{X}) \otimes_{\mathbb{Z}\left[\pi_{1}(X)\right]} P\right)$. Using the usual involution on group rings given by $\bar{g}=g^{-1}$ we can turn left $\mathbb{Z}[G]$-modules into right $\mathbb{Z}[G]$-modules. We define $H^{*}(X ; P):=H_{*}\left(\operatorname{Hom}_{\mathbb{Z}\left[\pi_{1}(X)\right]}\left(C_{*}(\tilde{X}), P\right)\right)$. 
Definition [31] Let $Y \subset X$ be CW-complexes, write $G:=\pi_{1}(X)$. Then $(X, Y)$ is called a Poincaré pair of dimension $n$ if there exists $[X] \in H_{n}(X)$, such that

$$
\begin{array}{rrrl}
\cap[X]: & H^{i}(X, Y ; \mathbb{Z}[G]) & \longrightarrow & H_{n-i}(X ; \mathbb{Z}[G]) \\
\cap \partial[X]: & H^{i}(Y ; \mathbb{Z}[G]) & \longrightarrow & H_{n-i-1}(Y ; \mathbb{Z}[G])
\end{array}
$$

are isomorphisms of $\mathbb{Z}[G]$-right modules. $[X]$ is called the fundamental homology class of $(X, Y)$.

Note that if $(X, Y)$ is a Poincaré complex, then from the long exact homology and cohomology sequences it follows that

$$
\cap[X]: H^{i}(X ; \mathbb{Z}[G]) \rightarrow H_{n-i}(X, Y ; \mathbb{Z}[G])
$$

is an isomorphism as well. In our case, $Y$ will be the 3 -manifold $M_{K}$ so that the second condition on $\partial[X]$ will be satisfied. Recall that we have an inclusion $\varphi: M_{K} \hookrightarrow K(G, 1)$ that induces an epimorphism of fundamental groups. For brevity we set

$$
M:=M_{K} \quad \text { and } \quad X:=K(G, 1) .
$$

Recall that if $(X, M)$ is a finite 4 -dimensional Poincaré pair, we say that $(M, \varphi)$ satisfies the Poincaré duality condition.

Lemma 3.2 Assume that $G$ satisfies $H_{3}(G)=0$ and $H^{i}(G ; \mathbb{Z}[G])=0$ for $i>2$. Then $(M, \varphi)$ satisfies the Poincaré duality condition if and only if

$$
\varphi^{*}: H^{i}(G ; \mathbb{Z}[G]) \longrightarrow H^{i}(M ; \mathbb{Z}[G])
$$

is an isomorphism for $i=1,2$.

Remark In Section 4 we will show that the above assumptions are satisfied for $G=S R$. In this case $H^{2}(G ; \mathbb{Z}[G]) \neq 0$ (cf Lemma 4.3) which shows that the Poincaré duality condition in general can not be simplified to $H^{2}\left(M_{K} ; \mathbb{Z}[G]\right)=$ 0 . Note also that the lemma implies that $H_{i}(G)=0$ for all $i \geq 3$.

Proof We first show the "only if" direction. In that case $H^{4-i}(X, M ; \mathbb{Z}[G]) \cong$ $H_{i}(X ; \mathbb{Z}[G])$. But $H_{i}(X ; \mathbb{Z}[G])=0$ for $i \neq 0$ since this is the homology of the $G$-cover of $X$ which is contractible since $X=K(G, 1)$. The claim now follows from the long exact cohomology sequence with $\mathbb{Z}[G]$-coefficients. We now turn to the proof of the "if" direction. By the long exact homology sequence of $(X, M)$, with $\mathbb{Z}$-coefficients, and the vanishing of $H_{3}(G)=H_{3}(X)$ we can choose a class $[X] \in H_{4}(X, M)$ that maps to the fundamental class $[M] \in$ $H_{3}(M)$ under the boundary map. Cap product with this class induces maps as 
in $(\cap)$ above and we need to check that they are isomorphisms. These maps are the left (or right) most vertical arrows in the commutative diagram of long exact sequences (with $\mathbb{Z}[G]$-coefficients understood):

$$
\begin{aligned}
& \rightarrow H^{i}(X, M) \rightarrow H^{i}(X) \quad \rightarrow \quad H^{i}(M) \quad \rightarrow \quad H^{i+1}(X, M) \\
& \downarrow \cap(-1)^{i}[X] \quad \downarrow \cap(-1)^{i}[X] \quad \downarrow \cap(-1)^{i+1}[M] \quad \downarrow \cap(-1)^{i+1}[X] \\
& \rightarrow H_{4-i}(X) \quad \rightarrow \quad H_{4-i}(X, M) \quad \rightarrow \quad H_{3-i}(M) \quad \rightarrow \quad H_{4-(i+1)}(X)
\end{aligned}
$$

Recall that $H_{i}(X ; \mathbb{Z}[G])=0$ for $i \neq 0$. Therefore, we need to show in particular that $H^{i}(X, M ; \mathbb{Z}[G])=0$ for $i \neq 4$. Note that

$$
H_{4-i}(X, M ; \mathbb{Z}[G]) \cong H_{3-i}(M ; \mathbb{Z}[G]) \quad \text { for } i \neq 3 .
$$

Since the maps $\cap[M]$ are isomorphisms it suffices to show that for all $i \neq 3$ the maps

$$
\varphi^{*}: H^{i}(X ; \mathbb{Z}[G]) \longrightarrow H^{i}(M ; \mathbb{Z}[G])
$$

are isomorphisms. For $i=1,2$ this is our assumption so it suffices to discuss the other cases. For $i=0$ both groups are zero since $G$ has infinite order. For $i>3$ again both groups are zero, for $X$ this is our assumption and for $M$ it follows from $\operatorname{dim} M=3$. Finally, we need to discuss the special case $i=3$ in the diagram above. Then

$$
H_{0}(M ; \mathbb{Z}[G]) \cong \mathbb{Z} \cong H_{0}(X ; \mathbb{Z}[G])
$$

and by assumption $H^{i}(X ; \mathbb{Z}[G])=0$ for $i=3,4$. It follows that the boundary map $H^{3}(M ; \mathbb{Z}[G]) \rightarrow H^{4}(X, M ; \mathbb{Z}[G])$ is an isomorphism and by commutativity of the diagram the last map in question

$$
\cap[X]: H^{4}(X, M ; \mathbb{Z}[G]) \longrightarrow H_{0}(X ; \mathbb{Z}[G])
$$

is also an isomorphism.

Lemma 3.3 Let $G$ satisfy $H_{3}(G)=0$ and $H^{i}(G ; \mathbb{Z}[G])=0$ for $i>2$. Furthermore, assume that $\operatorname{Hom}_{\mathbb{Z}[G]}\left(H_{i}(M ; \mathbb{Z}[G]), \mathbb{Z}[G]\right)=0$ for $i=1,2$. Then $(M, \varphi)$ satisfies the Poincaré duality condition if and only if the (Ext) condition from Theorem 1.3 holds: $\operatorname{Ext}_{\mathbb{Z}[G]}^{1}\left(H_{1}(M ; \mathbb{Z}[G]), \mathbb{Z}[G]\right)=0$.

Proof Denote the universal cover of $M$ by $\widetilde{M}$. Write $\pi:=\pi_{1}(M)$. Note that we have a chain isomorphism of right $\mathbb{Z}[G]$-module complexes given by

$$
\begin{aligned}
\operatorname{Hom}_{\mathbb{Z}[\pi]}\left(C_{*}(\widetilde{M}), \mathbb{Z}[G]\right) & \rightarrow \operatorname{Hom}_{\mathbb{Z}[G]}\left(C_{*}(\widetilde{M}) \otimes_{\mathbb{Z}[\pi]} \mathbb{Z}[G], \mathbb{Z}[G]\right) \\
\phi & \mapsto(c \otimes f \mapsto \overline{\phi(c)} f) .
\end{aligned}
$$


Therefore $H^{*}(M ; \mathbb{Z}[G]) \cong H_{*}\left(\operatorname{Hom}_{\mathbb{Z}[G]}\left(C_{*}(\widetilde{M}) \otimes_{\mathbb{Z}[\pi]} \mathbb{Z}[G], \mathbb{Z}[G]\right)\right)$. We now apply the universal coefficient spectral sequence (UCSS). This has an $E_{2}$-term

$$
E_{2}^{p, q}=\operatorname{Ext}_{\mathbb{Z}[G]}^{p}\left(H_{q}(M ; \mathbb{Z}[G]), \mathbb{Z}[G]\right),
$$

differentials $d_{r}$ of degree $(r, 1-r)$ and converges to

$$
H^{p+q}(M ; \mathbb{Z}[G]) \cong H_{p+q}\left(\operatorname{Hom}_{\mathbb{Z}[G]}\left(C_{*}(\widetilde{M}) \otimes_{\mathbb{Z}[\pi]} \mathbb{Z}[G], \mathbb{Z}[G]\right)\right),
$$

cf [20, Theorem 2.7] for more details. We use that the edge homomorphism at $q=0$ of the spectral sequence is the map

$$
\varphi^{*}: \operatorname{Ext}_{\mathbb{Z}[G]}^{p}\left(H_{0}(M ; \mathbb{Z}[G]), \mathbb{Z}[G]\right) \cong H^{p}(G ; \mathbb{Z}[G]) \longrightarrow H^{p}(M ; \mathbb{Z}[G]) .
$$

By Lemma 3.2 we only need to verify that this is an isomorphism for $p=1,2$ if and only if condition (Ext) holds. For $p=1$ this follows immediately from our assumption $\operatorname{Hom}_{\mathbb{Z}[G]}\left(H_{1}(M ; \mathbb{Z}[G]), \mathbb{Z}[G]\right)=0$. For $p=2$ we first observe that since $H^{3}(G ; \mathbb{Z}[G])=0$ there are no possible $d_{2}$-differentials into the $E_{2}^{1,1}$ spot. Since $\operatorname{Hom}_{\mathbb{Z}[G]}\left(H_{2}(M ; \mathbb{Z}[G]), \mathbb{Z}[G]\right)=0$, we get a short exact sequence

$$
0 \longrightarrow \operatorname{Ext}_{\mathbb{Z}[G]}^{1}\left(H_{1}(M ; \mathbb{Z}[G]), \mathbb{Z}[G]\right) \longrightarrow H^{2}(G ; \mathbb{Z}[G]) \stackrel{\varphi^{*}}{\longrightarrow} H^{2}(M ; \mathbb{Z}[G]) \longrightarrow 0
$$

showing that the condition (Ext) holds if and only if $\varphi^{*}$ is an isomorphism.

Let $G$ be any group with $H_{1}(G)=\mathbb{Z}$. Then we define $\Delta_{G}(t) \in \mathbb{Z}\left[t^{ \pm 1}\right]$ to be the order of the $\mathbb{Z}\left[t^{ \pm 1}\right]$-module $H_{1}\left(G, \mathbb{Z}\left[t^{ \pm 1}\right]\right) \cong H_{1}\left(G^{(1)}, \mathbb{Z}\right)$. Note that $\Delta_{G}$ is well-defined up to multiplication by a unit in $\mathbb{Z}\left[t^{ \pm 1}\right]$ and up taking the natural involution $t \mapsto t^{-1}$. For example if $G=S R$, then $\Delta_{G}(t)=t-2$.

Corollary 3.4 Let $G$ and $M$ satisfy all the conditions from Lemma 3.3, including the (Ext) condition. Then

$$
\Delta_{K}(t)=\Delta_{G}(t) \Delta_{G}\left(t^{-1}\right) .
$$

In particular $\operatorname{Arf}(K)=0$.

Proof By Lemma $3.3\left(K(G, 1), M_{K}\right)$ is a Poincaré pair. Since

$$
H_{i}\left(M_{K} ; \mathbb{Z}\right) \stackrel{\cong}{\rightrightarrows} H_{i}(K(G, 1) ; \mathbb{Z})
$$

for all $i$ it follows from a well-known argument that

$$
0=H_{i}\left(M_{K} ; \mathbb{Q}(t)\right) \stackrel{\cong}{\longrightarrow} H_{i}(K(G, 1) ; \mathbb{Q}(t))
$$

for all $i$. In particular $H_{i}\left(K(G, 1) ; \mathbb{Z}\left[t^{ \pm 1}\right]\right)$ is $\mathbb{Z}\left[t^{ \pm 1}\right]$-torsion for all $i$. The corollary now follows from the long exact sequence of $\left(K(G, 1), M_{K}\right)$ with $\mathbb{Z}\left[t^{ \pm 1}\right]-$ coefficients, duality, and the fact that the alternating product of orders of a 
long exact sequence equals one (cf [19, Lemma 5, p. 76]). The Arf invariant $\operatorname{Arf}(K) \in \mathbb{Z} / 2$ of a knot $K$ has the well-known property that it equals zero if $\Delta_{K}(-1) \equiv \pm 1 \bmod 8$, and 1 otherwise.

We shall now work through the remaining steps outlined in Section 3.1. We have a Poincaré pair $(X, M)$ where $X=K(G, 1)$ and $M=M_{K}$. Clearly $\pi_{1}(X)$ is normally generated by the image of a meridian for $K$ since $\varphi$ is surjective and since any meridian normally generates $\pi_{1}\left(M_{K}\right)$. Furthermore $H_{1}(X) \cong H_{1}(G) \cong \mathbb{Z}$ and $H_{2}(X)=H_{2}(G)=0$. It follows from the homology exact sequence of the pair $(X, M)$, Poincaré duality, and the fact that $\varphi$ is an isomorphism on $H_{1}$ that $H_{3}(X)=0$.

\subsection{The degree 1 normal map}

We show that the stable Spivak normal bundle of $(X, M)$ has a linear reduction. It seems well known to experts that this is always true in the oriented case in 4 dimensions but we could not find an explicit reference. So here is a rather ad hoc argument: Since $M$ is orientable, its tangent bundle is trivial and so is its stable normal bundle. We claim that the stable Spivak normal bundle of $X$ is also trivial: Let $B S$ be the classifying space of such (oriented) bundles. Its homotopy groups are the (shifted) stable homotopy groups of spheres, starting with

$$
\pi_{2} B S \cong \mathbb{Z} / 2 \cong \pi_{3} B S \text { and } \quad \pi_{4} B S \cong \mathbb{Z} / 24 .
$$

It follows that for all $i$ we have $H^{i}\left(X ; \pi_{i} B S\right)=0$ which shows that the classifying map $X \rightarrow B S$ of the stable Spivak normal bundle is trivial. Finally, it is clear that, up to homotopy, this trivial map can be lifted (trivially) through the fibration $B S O \rightarrow B S$, having the trivial map on the boundary $M$. From the usual transversality theory we obtain a degree 1 normal map from a manifold pair

$$
f:(N, M) \rightarrow(X, M)
$$

that is the identity on the boundary. Note that since the stable normal bundle of $X$ is trivial, so is that of $N$. In particular, $N$ is spin. Another approach to finding $f$ is to prove that $(M, \varphi)$ represents the zero element in the spin-bordism group $\Omega_{4}^{\text {Spin }}(X)$. The fact that this element is zero comes from comparing with the Poincaré duality spin bordism group in which $X$ itself gives a zero bordism. Even though this is not really important for our main argument, we next show that $f$ is unique in a certain sense: In the spin case the normal cobordism group of degree 1 normal maps is isomorphic to

$$
H_{2}(X ; \mathbb{Z} / 2) \oplus 8 \cdot \mathbb{Z}
$$


where the $8 \cdot \mathbb{Z}$ summand is the difference of ordinary signatures, $\sigma(N)-\sigma(X)$. The first summand vanishes in our case and so does $\sigma(X)$. By adding copies of Freedman's $E_{8}$-manifold to $N$, we may assume that $\sigma(N)=0$. This is just another way of saying that we work with the reduced normal cobordism group.

\subsection{The surgery obstruction}

There is a well defined surgery obstruction $\sigma(f)$ in $L_{4}^{h}(\mathbb{Z}[G])$ which in our case is simply the intersection form on $\pi_{2} N$. The first step is 'surgery below the middle dimension' which makes $f 2$-connected. The surgery obstruction then measures the kernel of $f$ in the middle dimension which in our case is in $\pi_{2}$. To make this precise, recall the following definition from [31, p. 47].

Definition A quadratic form over $\mathbb{Z}[G]$ is defined to be a triple $(H, \lambda, \mu)$ with $H$ a free $\mathbb{Z}[G]$-module,

$$
\lambda: H \times H \rightarrow \mathbb{Z}[G]
$$

a non-singular hermitian form and

$$
\mu: H \rightarrow \mathbb{Z}[G] /\langle a-\bar{a} \mid a \in \mathbb{Z}[G]\rangle
$$

a quadratic refinement. Here a form is non-singular if the induced map $H \rightarrow$ $\operatorname{Hom}_{\mathbb{Z}[G]}(H ; \mathbb{Z}[G])$ is an isomorphism. A form isomorphic to a direct sum of the form $(\mathbb{Z}[G] \cdot e \oplus \mathbb{Z}[G] \cdot f, \lambda, \mu)$, where $\lambda(e, f)=1$ and $\mu(e)=\mu(f)=0$, is called a hyperbolic form.

It should be pointed out that in the oriented case, where the involution on $\mathbb{Z}[G]$ is given by $\bar{g}:=g^{-1}$, the quadratic refinement $\mu$ is completely determined by the hermitian form $\lambda$. Its only role is to make sure that $\lambda$ is even in the sense that for every $h \in H$ there is an $m \in \mathbb{Z}[G]$ such that

$$
\lambda(h, h)=m+\bar{m}
$$

The main examples of hermitian forms come from the intersection form of 4-manifolds, where $G=\pi_{1} W$ and $(H, \lambda)=\left(\pi_{2} W^{4}, \lambda_{W}\right)$. If $h \in \pi_{2} W$ is represented by an immersed 2-sphere $S$ in $W$, then one can look at the selfintersections $m(S)$ that are related to the intersection $\lambda_{W}$ of $S$ and a parallel push-off by the following formula:

$$
\lambda_{W}(S, S)=m(S)+\bar{m}(S)+e(S) \cdot 1
$$

Here $e(S) \in \mathbb{Z}$ denotes the normal Euler class of $S$. Note that the coefficient of $1 \in G$ in $m(S)$ can be changed arbitrarily by a (nonregular) homotopy, the so 
called cusp move. This also changes $e(S)$ so as to make the above formula still hold (the left hand side only depends on the homotopy class of $S$ ). It follows that the intersection form $\lambda$ on $\pi_{2}$ of a 4 -manifold is even in the above sense if and only if $e(S)$ is an even integer, ie, the second Stiefel-Whitney class $w_{2}(S)$ vanishes. That is why we will completely ignore the quadratic refinement $\mu$ in the following, only making sure that our manifolds are spin. Note that for general spin 4-manifolds $W$ with fundamental group $G$, the intersection form $\lambda_{W}$ on $\pi_{2} W$ is not a quadratic form in the above sense. The problems are that in general $\pi_{2} W$ is not free and $\lambda_{W}$ is not non-singular. However, given a 2-connected degree 1 map $f: N \rightarrow X$, one can restrict $\lambda_{N}$ to the kernel of $f$ on $\pi_{2}$. Then both of these conditions can be arranged after stabilizing $N$ by copies of $S^{2} \times S^{2}$, 31, p. 26]. Moreover, if $w_{2}$ vanishes on 2-spheres in this kernel then a quadratic refinement exists by the above considerations. If $f$ is a normal map (in addition to having degree 1) then this last condition is obvious. In our setting, $\pi_{2} K(G, 1)=0$ so that we automatically work on the kernel of $f$. Moreover, we saw that $N$ is spin so that the intersection form $\lambda_{N}$ on $\pi_{2} N$ is even and hence represents a unique quadratic form, up to the stabilization with hyperbolic forms. This motivates the following definition. Consider the semigroup of quadratic forms under direct sum. We say that forms $X_{1}, X_{2}$ are equivalent if there exist hyperbolic forms $H_{1}, H_{2}$, such that $X_{1} \oplus H_{1}$ and $X_{2} \oplus H_{2}$ are isomorphic. The set of equivalence classes form a group (cf [27, p. 249]), denoted by $L_{4}^{h}(\mathbb{Z}[G])$. As discussed above, $\left(\pi_{2} N, \lambda_{N}\right)$ represents an element in this group.

Theorem 3.5 The ordinary signature $\sigma$ induces isomorphisms

(1) $\quad L_{4}^{h}(\mathbb{Z}) \stackrel{\cong}{\longrightarrow} \mathbb{Z}, \quad(H, \lambda, \mu) \mapsto \sigma(\lambda) / 8$

(2) $\quad L_{4}^{h}(\mathbb{Z}[G]) \stackrel{\cong}{\longrightarrow} L_{4}^{h}(\mathbb{Z})$ for $G=\mathbb{Z}$ or $G=S R$.

The first statement is well-known, see for example [31, Theorem 13A.1]. The second statement is well-known in the case that $G=\mathbb{Z}$ (cf [26]). We prove the case $G=S R$ in Section 4, In general if $G$ is a ribbon group, then $L_{4}^{h}(\mathbb{Z}[G]) \rightarrow$ $L_{4}^{h}(\mathbb{Z})$ is an isomorphism if the Whitehead conjecture and the Farrell--Jones conjecture hold. This has been shown in many interesting cases, cf [1] for the case of knot groups. By the computations in Section 3.3. the surgery obstruction $\sigma(f)=\lambda_{N}$ actually only depends on the original data $(M, \varphi)$ if we assume that $\sigma(N)=0$, ie, that it lies in the reduced L-group

$$
\sigma(M, \varphi)=\sigma(f)=\lambda_{N} \in \widetilde{L}_{4}^{h}(\mathbb{Z}[G]):=\operatorname{ker}\left(L_{4}^{h}(\mathbb{Z}[G]) \longrightarrow L_{4}^{h}(\mathbb{Z})\right)
$$

This element is hence an obstruction for finding a h-ribbon for $K$ with epimorphism $\phi: \pi_{1} M \rightarrow G$. If the reduced L-group vanishes then, after further 
stabilization by $S^{2} \times S^{2}$, we may assume that $\pi_{2} N$ has hyperbolic intersection form.

\subsection{Constructing the $\mathrm{h}-$-ribbon disk}

The assumptions of Theorem 1.9 say that the reduced L-group vanishes and that the topological surgery sequence $(\mathcal{S})$ is exact for our fundamental group. Therefore, one gets a topological manifold $W$ together with a homotopy equivalence $\left(W, M_{K}\right) \rightarrow\left(K(G, 1), M_{K}\right)$. Hence we found our h-ribbon by Proposition 3.1 and we finished the proof of Theorem 1.9.

\subsection{Proof of Theorem 1.3}

To construct a ribbon disk we use Theorem [1.9 that we just finished proving. For $G=S R$, we will show all the required properties in Section 4 . For $G=\mathbb{Z}$ they are easy to check, for example the conditions on Hom follow from the fact that the Alexander module $H_{1}\left(M_{K} ; \mathbb{Z}[\mathbb{Z}]\right)$ is a $\mathbb{Z}[\mathbb{Z}]$-torsion module. Moreover,

$$
\operatorname{Ext}_{\mathbb{Z}[\mathbb{Z}]}^{1}\left(H_{1}\left(M_{K} ; \mathbb{Z}[\mathbb{Z}]\right), \mathbb{Z}[\mathbb{Z}]\right) \cong H_{1}\left(M_{K} ; \mathbb{Z}[\mathbb{Z}]\right)
$$

so that our (Ext) condition simply means that the Alexander module (or, equivalently, the Alexander polynomial) of $K$ is trivial. Conversely, assume that there exists a h-ribbon $D$ for $K$ with $\pi_{1}\left(N_{D}\right) \cong G$. Then it follows from arguments as in the proof of Theorem 4.4 in [5] that the Blanchfield form $B \ell(G)$ always vanishes on

$$
\operatorname{Ker}\left\{H_{1}\left(M_{K} ; \mathbb{Z}[G]\right) \longrightarrow H_{1}\left(N_{D} ; \mathbb{Z}[G]\right)\right\},
$$

In our case this group equals $H_{1}\left(M_{K} ; \mathbb{Z}[G]\right)$ since $H_{1}\left(N_{D} ; \mathbb{Z}[G]\right)=0$. In the Section [5 we will recall this Blanchfield form and show in Lemma 5.1 that its vanishing is indeed equivalent to our (Ext) condition.

\section{Properties of the solvable ribbon group $S R$}

The purpose of this section is to prove that the assumptions of Lemma 3.3 are satisfied for the group $G=S R$ :

Lemma 4.1 The group $G=S R$ satisfies $H_{3}(G)=0$ and $H^{i}(G ; \mathbb{Z}[G])=0$ for $i>2$. Furthermore, for any knot $K$ and any epimorphism $\pi_{1}\left(M_{K}\right) \rightarrow G$ one has

$$
\operatorname{Hom}_{\mathbb{Z}[G]}\left(H_{i}\left(M_{K} ; \mathbb{Z}[G]\right), \mathbb{Z}[G]\right)=0 \quad \text { for } \quad i=1,2
$$


A group $G$ is called poly-torsion-free-abelian (PTFA) if there exists a filtration

$$
1=G_{0} \triangleleft G_{1} \triangleleft \cdots \triangleleft G_{n-1} \triangleleft G_{n}=G
$$

such that $G_{i} / G_{i-1}$ is torsion free abelian. As we pointed out in Section 2 the only solvable ribbon groups are $\mathbb{Z}$ and $S R$ and clearly both are PTFA. If $G$ is a PTFA group then the group ring $\mathbb{Z}[G]$ satisfies the Ore condition and therefore has an Ore localization, cf [5, Proposition 2.5]

$$
Q(G):=\mathbb{Z}[G](\mathbb{Z}[G] \backslash 0)^{-1}
$$

which is a skew field. Therefore, every $Q(G)$-module is free and its rank is well-defined. As a localization, $Q(G)$ is flat as a right (and left) $\mathbb{Z}[G]$-module. Moreover, the $\mathbb{Z}[G]$-torsion part of a module is a submodule (unlike for general non-commutative rings). Let $K$ be a knot and $\pi_{1}\left(M_{K}\right) \rightarrow G$ be an epimorphism onto a PTFA group. From [5, Proposition 2.11] it follows that

$$
H_{1}\left(M_{K} ; \mathbb{Z}[G]\right) \otimes_{\mathbb{Z}[G]} Q(G) \cong H_{1}\left(M_{K} ; Q(G)\right)=0,
$$

ie, $H_{1}\left(M_{K} ; \mathbb{Z}[G]\right)$ is $\mathbb{Z}[G]$-torsion. In particular, the required condition

$$
\operatorname{Hom}_{\mathbb{Z}[G]}\left(H_{1}\left(M_{K} ; \mathbb{Z}[G]\right), \mathbb{Z}[G]\right)=0
$$

in Lemma 4.1 follows. Furthermore,

$$
H_{0}\left(M_{K} ; Q(G)\right) \cong H_{0}\left(M_{K} ; \mathbb{Z}[G]\right) \otimes_{\mathbb{Z}[G]} Q(G) \cong \mathbb{Z} \otimes_{\mathbb{Z}[G]} Q(G)=0
$$

and similarly $H_{3}\left(M_{K} ; Q(G)\right)=0$ since $H_{3}\left(M_{K} ; \mathbb{Z}[G]\right)=0$. The Euler characteristics of $M_{K}$ with $\mathbb{Z}$ and $Q(G)$ coefficients agree by the usual argument and hence

$$
\chi_{Q(G)}\left(H_{*}\left(M_{K} ; Q(G)\right)\right)=\chi_{\mathbb{Z}}\left(H_{*}\left(M_{K} ; \mathbb{Z}\right)\right)=0 .
$$

It follows that

$$
H_{2}\left(M_{K} ; \mathbb{Z}[G]\right) \otimes_{\mathbb{Z}[G]} Q(G) \cong H_{2}\left(M_{K} ; Q(G)\right)=0,
$$

Therefore $H_{1}\left(M_{K} ; Q(G)\right)=0$ and hence $\operatorname{Hom}_{\mathbb{Z}[G]}\left(H_{2}\left(M_{K} ; \mathbb{Z}[G]\right), \mathbb{Z}[G]\right)=0$ because $H_{2}\left(M_{K} ; \mathbb{Z}[G]\right)$ is again $\mathbb{Z}[G]$-torsion. So another required condition in Lemma 4.1 follows:

$$
\operatorname{Hom}_{\mathbb{Z}[G]}\left(H_{2}\left(M_{K} ; \mathbb{Z}[G]\right), \mathbb{Z}[G]\right)=0 .
$$

The following result finishes up our task of showing $S R$ has all the required properties.

Lemma 4.2 The group $G=S R$ is aspherical, in particular $\operatorname{Ext}_{\mathbb{Z}[G]}^{i}(\mathbb{Z}, \mathbb{Z}[G])=$ 0 for $i>2$ and $H_{3}(G)=0$. 
Proof Let $D$ be a ribbon disk with complement $N_{D}$ and fundamental group $\pi_{1}\left(N_{D}\right)=G=S R$. Recall that by Alexander duality $H_{2}\left(N_{D}\right)=0$. We will show that $N_{D}$ is a $K(G, 1)$. First note that $N_{D}$ is homotopy equivalent to a 2-complex $N$. By the Hurewicz theorem it suffices to prove the vanishing of $\pi_{2}(N) \cong H_{2}(\tilde{N})$. Let $Q(G)$ be the Ore localization of $\mathbb{Z}[G]$. By the same arguments as above for $M_{K}$, involving Euler characteristics, one shows that

$$
H_{2}(\tilde{N}) \otimes_{\mathbb{Z}[G]} Q(G) \cong H_{2}(N ; \mathbb{Z}[G]) \otimes_{\mathbb{Z}[G]} Q(G)=0 .
$$

Now consider the following commutative diagram

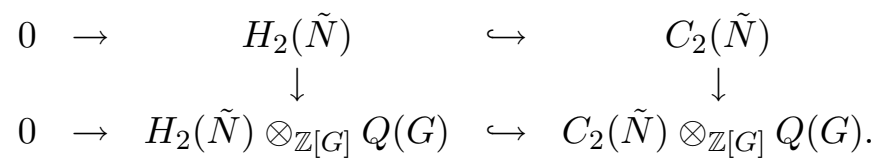

The second vertical map is injective since $C_{2}(\tilde{N})$ is a free $\mathbb{Z}[G]$-module, hence the first vertical map is injective as well, hence $H_{2}(\tilde{N})=0$.

Remark We could have used the well known fact that a one-relator group is aspherical if the relation is not a proper power (so in particular the group is torsion free). However, a similar argument as above will also be used in the proof of Lemma 4.3. Locally indicable ribbon groups are also known to be aspherical.

The following result is not needed in the rest of the paper but we include it for the interested reader. The nontriviality of the homology group in question made it clear that some more naive formulations of the Poincaré duality condition must fail. Recall from Section 2 that $S R$ has the presentation

$$
S R=\left\langle a, c \mid a c a^{-1}=c^{2}\right\rangle .
$$

Lemma 4.3 Let $G:=S R$, then $H^{2}(G ; \mathbb{Z}[G])$ maps onto $\mathbb{Z}[1 / 2]$. More precisely, as a right $\mathbb{Z}[G]$-module, $H^{2}(G ; \mathbb{Z}[G])$ is the quotient of $\mathbb{Z}[G]$ by the right ideal generated by

$$
1-a c a^{-1} \text { and } a-a c a^{-1} c^{-1}-a c a^{-1} c^{-2} .
$$

Dividing $\mathbb{Z}[G]$ by the two-sided ideal generated by the same elements gives the ring $\mathbb{Z}[1 / 2]$.

Proof The proof of Lemma 4.1 shows that the 2-complex associated to the above presentation is a $K(G, 1)$. Therefore, we can calculate $H^{2}(G ; \mathbb{Z}[G])$ from the short resolution

$$
0 \longrightarrow \mathbb{Z}[G] \longrightarrow \mathbb{Z}[G]^{2} \longrightarrow \mathbb{Z}[G] \stackrel{\epsilon}{\longrightarrow} \mathbb{Z} \longrightarrow 0
$$


where the first map is given by the Fox derivatives $\partial_{a}$ and $\partial_{c}$ of the relation $a c a^{-1} c^{-2}$. A straightforward calculation using Fox derivatives 'from the left' gives

$$
\partial_{a}=1-a c a^{-1} \quad \text { and } \quad \partial_{c}=a-a c a^{-1} c^{-1}-a c a^{-1} c^{-2}
$$

If we now decide to quotient by the two-sided ideal generated by the first relation, we find that the defining relation forces $c=1$ and hence we are left with $\mathbb{Z}[\mathbb{Z}]$, generated by $a$. The second relation then introduces the relation $a=2$ into this commutative ring which gives the ring $\mathbb{Z}[1 / 2]$.

\section{Computation of $L_{4}^{h}(\mathbb{Z}[S R])$}

Lemma 4.4 The inclusion map $\mathbb{Z} \rightarrow \mathbb{Z}[\mathbb{Z} \ltimes \mathbb{Z}[1 / 2]]$ induces an isomorphism $L_{4}^{h}(\mathbb{Z}) \rightarrow L_{4}^{h}(\mathbb{Z}[\mathbb{Z} \ltimes \mathbb{Z}[1 / 2]])$.

In order to prove this lemma, we need a theorem by Ranicki, which in turn needs the notion of $L_{n}$-groups for any $n$. We refer to [25] for the definition of these groups. We recall that the Whitehead group $\mathrm{Wh}(G)$ for a group $G$ is defined as $\mathrm{Wh}(G)=K_{1}(\mathbb{Z}[G]) / \pm G\left(\operatorname{cf}\left[27\right.\right.$, p. 172] for a definition of $\left.K_{1}(\mathbb{Z}[G])\right)$.

Theorem 4.5 24] Let $G$ be a group with $\mathrm{Wh}(G)=0$ and let $\alpha: \mathbb{Z}[G] \rightarrow$ $\mathbb{Z}[G]$ be an automorphism, then there exists a long exact sequence

$$
\cdots \rightarrow L_{n}(\mathbb{Z}[G]) \stackrel{1-\alpha_{*}}{\longrightarrow} L_{n}(\mathbb{Z}[G]) \rightarrow L_{n}\left(\mathbb{Z}[G]^{\alpha}\left[t, t^{-1}\right]\right) \rightarrow L_{n-1}(\mathbb{Z}[G]) \rightarrow \ldots,
$$

where $\mathbb{Z}[G]^{\alpha}\left[t, t^{-1}\right]$ denotes the twisted Laurent ring, ie, for $a_{1}, a_{2} \in \mathbb{Z}[G]$ we have $a_{1} t^{n_{1}} \cdot a_{2} t^{n_{2}}=a_{1} \alpha\left(a_{2}\right)^{n_{1}} t^{n_{1}+n_{2}}$.

Remark Since $W h(G)=0$, we don't have to distinguish between $L^{s}$ and $L^{h}$. We will therefore henceforth drop any decorations.

We'll make use of the fact that $\mathbb{Z}[1 / 2]$ is the direct limit of the direct system $\mathbb{Z} \stackrel{\cdot 2}{\rightarrow} \mathbb{Z} \stackrel{\cdot 2}{\rightarrow} \ldots$. We'll therefore write $\mathbb{Z}[1 / 2]=\lim \mathbb{Z}$. Denote the map $\cdot 2: \mathbb{Z}[1 / 2] \rightarrow \mathbb{Z}[1 / 2]$ by $\alpha$; then $\mathbb{Z}[\mathbb{Z} \ltimes \mathbb{Z}[1 / 2]] \cong \mathbb{Z}[\mathbb{Z}[1 / 2]]^{\alpha}\left[t, t^{-1}\right]$.

Claim $\operatorname{Wh}(\mathbb{Z}[1 / 2])=0$.

The determinant map det: $K_{1}(\mathbb{Z}[\mathbb{Z}]) \rightarrow \mathbb{Z} \times\{ \pm 1\}$ is an isomorphism (cf [27, p. 172]). The $K_{1}$ functor commutes with direct limits and $\alpha$ commutes with the determinant maps, hence det: $K_{1}(\mathbb{Z}[\mathbb{Z}[1 / 2]]) \rightarrow \mathbb{Z}[\mathbb{Z}[1 / 2]] \times\{ \pm 1\}$ is an isomorphism as well. It now follows immediately that $\mathrm{Wh}(\mathbb{Z}[1 / 2])=0$. 
By Theorem 4.5 there therefore exists an exact sequence

$$
\begin{array}{rll}
\cdots & \rightarrow L_{n}(\mathbb{Z}[\mathbb{Z}[1 / 2]]) & \stackrel{1-\alpha_{*}}{\longrightarrow} L_{n}(\mathbb{Z}[\mathbb{Z}[1 / 2]]) \rightarrow L_{n}\left(\mathbb{Z}[\mathbb{Z}[1 / 2]]^{\alpha}\left[t, t^{-1}\right]\right) \rightarrow \\
& \rightarrow L_{n-1}(\mathbb{Z}[\mathbb{Z}[1 / 2]]) & \stackrel{1-\alpha_{*}}{\longrightarrow} \ldots
\end{array}
$$

It is clear that the proposition follows once we prove the following claim.

Claim (1) $\quad L_{4}(\mathbb{Z}) \rightarrow L_{4}(\mathbb{Z}[\mathbb{Z}[1 / 2]]$ is an isomorphism,

(2) $\quad \alpha_{*}: L_{4}(\mathbb{Z}[\mathbb{Z}[1 / 2]]) \rightarrow L_{4}(\mathbb{Z}[\mathbb{Z}[1 / 2]])$ is the identity map,

(3) $\quad L_{3}(\mathbb{Z}[\mathbb{Z}[1 / 2]])=0$.

We recall that (cf 31] )

$$
L_{n}(\mathbb{Z})=\left\{\begin{array}{rlll}
0 & \text { if } & n \equiv 1 & \bmod 2 \\
\mathbb{Z} & \text { if } & n \equiv 0 & \bmod 4 \\
\mathbb{Z} / 2 & \text { if } & n \equiv 2 & \bmod 4
\end{array}\right.
$$

From Theorem 4.5 it follows that $\left.L_{4}(\mathbb{Z}]\right) \stackrel{\cong}{\rightrightarrows} L_{4}(\mathbb{Z}[\mathbb{Z}])$ via the map induced by the inclusion $\mathbb{Z} \rightarrow \mathbb{Z}[\mathbb{Z}]$. The inverse $L_{4}(\mathbb{Z}[\mathbb{Z}]) \rightarrow L_{4}(\mathbb{Z})$ is given by tensoring with $\mathbb{Z}$, considered as a $\mathbb{Z}[\mathbb{Z}]$-module via the map $t \mapsto 1$. In particular the map $\mathbb{Z} \stackrel{\cdot 2}{\longrightarrow} \mathbb{Z}$ induces the identity on the $L_{4}$ group of the group ring, ie, on $L_{4}(\mathbb{Z}[\mathbb{Z}])$. From the fact that the $L$-functor commutes with direct limits, we immediately get the first two statements. From [31, p. 181] we get the following commutative diagram

$$
\begin{array}{cccc}
L_{3}(\mathbb{Z}[\mathbb{Z}]) & \rightarrow & L_{3}(\mathbb{Z}[\mathbb{Z} / 2]) \\
\alpha_{*} \downarrow & & \downarrow \alpha_{*} \\
L_{3}(\mathbb{Z}[\mathbb{Z}]) & \rightarrow & L_{3}(\mathbb{Z}[\mathbb{Z} / 2]),
\end{array}
$$

where $\alpha$ denotes multiplication by two. In particular $\alpha_{*}: L_{3}(\mathbb{Z}[\mathbb{Z} / 2]) \rightarrow$ $L_{3}(\mathbb{Z}[\mathbb{Z} / 2])$ factors through $L_{3}(\mathbb{Z})=0$. Hence $\alpha_{*}: L_{3}(\mathbb{Z}[\mathbb{Z}]) \rightarrow L_{3}(\mathbb{Z}[\mathbb{Z}])$ is the zero map, taking direct limits we see that $L_{3}(\mathbb{Z}[\mathbb{Z}[1 / 2]])=0$. This completes the proof of Lemma 4.4 .

\section{Non-commutative Blanchfield forms}

Let $G$ be a PTFA-group and $\varphi: M_{K} \rightarrow G$ a homomorphism. Note that the involution on $\mathbb{Z}[G]$ extends to an involution on $Q(G)$ and on $Q(G) / \mathbb{Z}[G]$. Let $\pi:=\pi_{1}\left(M_{K}\right)$. Note that the map

$$
\begin{aligned}
\operatorname{Hom}_{\mathbb{Z}[\pi]}\left(C_{*}\left(\tilde{M}_{K}\right), Q(G) / \mathbb{Z}[G]\right) & \rightarrow \operatorname{Hom}_{\mathbb{Z}[G]}\left(C_{*}\left(\tilde{M}_{K}\right) \otimes_{\mathbb{Z}[\pi]} \mathbb{Z}[G], Q(G) / \mathbb{Z}[G]\right) \\
\phi & \mapsto(c \otimes f \mapsto \overline{\phi(c)} f)
\end{aligned}
$$


is well-defined and induces an evaluation map

$$
H^{1}\left(M_{K} ; Q(G) / \mathbb{Z}[G]\right) \longrightarrow \operatorname{Hom}_{\mathbb{Z}[G]}\left(H_{1}\left(M_{K} ; \mathbb{Z}[G]\right), Q(G) / \mathbb{Z}[G]\right) .
$$

Now consider

$$
\begin{aligned}
H_{1}\left(M_{K} ; \mathbb{Z}[G]\right) \stackrel{\cong}{\longrightarrow} H^{2}\left(M_{K} ; \mathbb{Z}[G]\right) \cong H^{1}\left(M_{K} ; Q(G) / \mathbb{Z}[G]\right) \\
\longrightarrow \operatorname{Hom}_{\mathbb{Z}[G]}\left(H_{1}\left(M_{K} ; \mathbb{Z}[G]\right), Q(G) / \mathbb{Z}[G]\right)
\end{aligned}
$$

ie, the composition of Poincaré duality, the inverse of the Bockstein homomorphism for the coefficient sequence $0 \rightarrow \mathbb{Z}[G] \rightarrow Q(G) \rightarrow Q(G) / \mathbb{Z}[G] \rightarrow 0$ and the above evaluation map.

Note that the second map is an isomorphism since the homology and hence the cohomology with $Q(G)$ coefficients vanishes. This map defines the hermitian Blanchfield pairing, of [8, Theorem 5.1]

$$
B \ell(G): H_{1}\left(M_{K} ; \mathbb{Z}[G]\right) \times H_{1}\left(M_{K} ; \mathbb{Z}[G]\right) \longrightarrow Q(G) / \mathbb{Z}[G] .
$$

Lemma 5.1 For $G=\mathbb{Z}$ or $S R$, the condition (Ext) from Theorem 1.3 is equivalent to the vanishing of the Blanchfield form $B \ell(G)$.

The remark after Lemma 3.2 shows that the vanishing of the Blanchfield pairing $B \ell(G)$ is a weaker statement than the vanishing of the corresponding homology group $H_{1}\left(M_{K} ; \mathbb{Z}[G]\right) \cong H^{2}\left(M_{K} ; \mathbb{Z}[G]\right)$. In particular, this shows that $B \ell(G)$ will in general be singular.

Proof of Lemma 5.1] From the long exact Ext-sequence corresponding to

$$
0 \longrightarrow \mathbb{Z}[G] \longrightarrow Q(G) \longrightarrow Q(G) / \mathbb{Z}[G] \longrightarrow 0
$$

it follows that

$$
\begin{aligned}
\operatorname{Ext}_{\mathbb{Z}[G]}^{1}\left(H_{1}\left(M_{K} ; \mathbb{Z}[G]\right), \mathbb{Z}[G]\right) & \cong \operatorname{Ext}_{\mathbb{Z}[G]}^{0}\left(H_{1}\left(M_{K} ; \mathbb{Z}[G]\right), Q(G) / \mathbb{Z}[G]\right) \\
& \cong \operatorname{Hom}_{\mathbb{Z}[G]}\left(H_{1}\left(M_{K} ; \mathbb{Z}[G]\right), Q(G) / \mathbb{Z}[G]\right)
\end{aligned}
$$

since $\operatorname{Ext}_{\mathbb{Z}[G]}^{i}\left(H_{1}\left(M_{K} ; \mathbb{Z}[G]\right), Q(G)\right)=0$ for all $i$ (cf [5, Remark 2.8]). We are now done once we show that the homomorphism

$$
H^{1}\left(M_{K} ; Q(G) / \mathbb{Z}[G]\right) \longrightarrow \operatorname{Hom}_{\mathbb{Z}[G]}\left(H_{1}\left(M_{K} ; \mathbb{Z}[G]\right), Q(G) / \mathbb{Z}[G]\right)
$$

in the definition of $B \ell(G)$ is surjective. As in the proof of Lemma 3.3 we have an isomorphism

$$
H^{1}\left(M_{K} ; Q(G) / \mathbb{Z}[G]\right) \cong H_{1}\left(\operatorname{Hom}_{\mathbb{Z}[G]}\left(C_{*}\left(\tilde{M}_{K}\right) \otimes_{\mathbb{Z}\left[\pi_{1}(M)\right]} \mathbb{Z}[G], Q(G) / \mathbb{Z}[G]\right)\right) .
$$


Now applying the UCSS we see that the evaluation map (5.2) is surjective if $\operatorname{Ext}_{\mathbb{Z}[G]}^{2}(\mathbb{Z}, Q(G) / \mathbb{Z}[G])=0$. Let $X=K(S R, 1)$. By Lemma 4.2 we can assume that $X$ is a $2-$ complex. Then

$$
\operatorname{Ext}_{\mathbb{Z}[G]}^{2}(\mathbb{Z}, Q(G) / \mathbb{Z}[G]) \cong H^{2}(G ; Q(G) / \mathbb{Z}[G]) \cong H^{2}(X ; Q(G) / \mathbb{Z}[G]) .
$$

Note that $H^{i}(X ; Q(G)) \cong H_{i}(X ; Q(G)) \cong H_{i}(X ; \mathbb{Z}[G]) \otimes_{\mathbb{Z}[G]} Q(G)=0$ for $i=2,3$, in particular it follows that

$$
H^{2}(X ; Q(G) / \mathbb{Z}[G])=H^{3}(X ; \mathbb{Z}[G])=0
$$

because $X$ is a $2-$ complex.

Since $B \ell(\mathbb{Z})$ is nonsingular for any knot $K$, it vanishes if and only if the Alexander module itself vanishes. This in turn is equivalent to $K$ having trivial Alexander polynomial and to our condition (Ext) for $G=\mathbb{Z}$.

\section{The satellite construction}

Let $K, C$ be knots. Let $A \subset S^{3} \backslash K$ be a curve, unknotted in $S^{3}$. Then $S^{3} \backslash \nu A$ is a solid torus. Let $\psi: \partial(\overline{\nu A}) \rightarrow \partial(\overline{\nu C})$ be a diffeomorphism which sends a meridian of $A$ to a longitude of $C$, and a longitude of $A$ to a meridian of $C$. The space

$$
\left(S^{3} \backslash \nu A\right) \cup_{\psi}\left(S^{3} \backslash \nu C\right)
$$

is a 3 -sphere and the image of $K$ is denoted by $S=S(K, C, A)$. We say $S$ is the satellite knot with companion $C$, orbit $K$ and axis $A$. Note that we replaced a tubular neighborhood of $C$ by a knot in a solid torus, namely $K \subset S^{3} \backslash \nu A$. Figure 6.1 shows that taking the Whitehead double of a knot is an example for the satellite construction. It is easy to see that

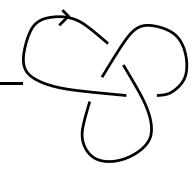

C

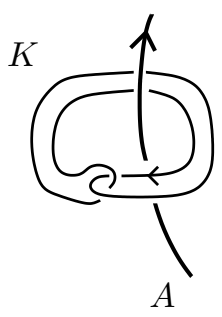

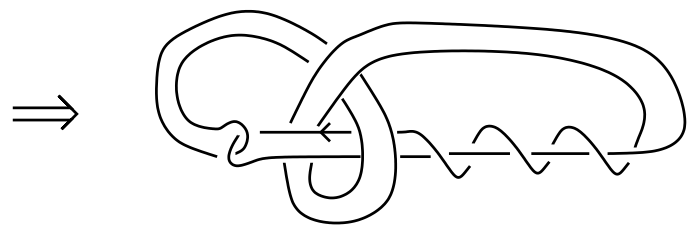

$S$

Figure 6.1: Satellite construction with $C$ the trefoil, $K$ the unknot

$$
M_{S}=\left(M_{K} \backslash \nu A\right) \cup_{\partial(\overline{\nu A})}\left(S^{3} \backslash \nu C\right)
$$


Obstruction theory shows that the map $\psi^{-1}: \partial(\overline{\nu C}) \rightarrow \partial(\overline{\nu A})$ can be extended to a map $f: S^{3} \backslash \nu C \rightarrow \overline{\nu A}$. Combining with the injection $M_{K} \backslash \nu A \rightarrow M_{K}$ this defines a map $M_{S} \rightarrow M_{K}$. Let $\varphi: \pi_{1}\left(M_{K}\right) \rightarrow G$ be a homomorphism onto a torsion-free group. Then we get an induced map $\pi_{1}\left(M_{S}\right) \rightarrow G$.

Lemma 6.2 If $\varphi(A)=e$, then $H_{1}\left(M_{S} ; \mathbb{Z}[G]\right) \cong H_{1}\left(M_{K} ; \mathbb{Z}[G]\right)$, otherwise

$$
H_{1}\left(M_{S} ; \mathbb{Z}[G]\right) \cong H_{1}\left(M_{K} ; \mathbb{Z}[G]\right) \oplus H_{1}\left(M_{C} ; \mathbb{Z}[\mathbb{Z}]\right) \otimes_{\mathbb{Z}[\mathbb{Z}]} \mathbb{Z}[G],
$$

where $\mathbb{Z}[G]$ is a $\mathbb{Z}[\mathbb{Z}] \cong \mathbb{Z}\left[t, t^{-1}\right]$ module via $t \mapsto \varphi(A)$.

Proof Consider the following commutative diagram of Meyer-Vietoris exact sequences (with $\mathbb{Z}[G]$-coefficients understood)

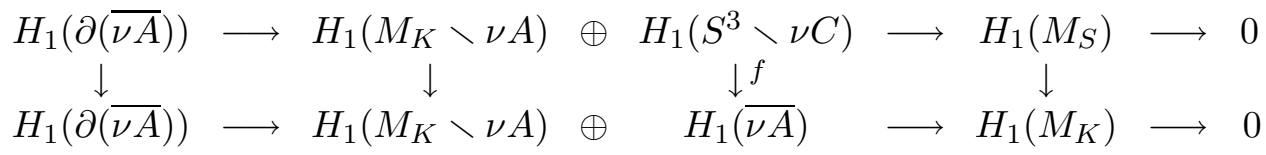

If $\varphi(A)=e$, then the coefficient systems for $\overline{\nu A}$ and for $S^{3} \backslash \nu C$ are trivial, hence

$$
\begin{aligned}
H_{1}(\overline{\nu A} ; \mathbb{Z}[G]) & \cong H_{1}(\overline{\nu A} ; \mathbb{Z}) \otimes_{\mathbb{Z}} \mathbb{Z}[G] \\
& \cong \mathbb{Z} \otimes_{\mathbb{Z}}[G] \\
& \cong H_{1}\left(S^{3} \backslash \nu C ; \mathbb{Z}\right) \otimes_{\mathbb{Z}} \mathbb{Z}[G] \\
& \cong H_{1}\left(S^{3} \backslash \nu C ; \mathbb{Z}[G]\right) .
\end{aligned}
$$

This immediately implies that $H_{1}\left(M_{S} ; \mathbb{Z}[G]\right) \cong H_{1}\left(M_{K} ; \mathbb{Z}[G]\right)$. If $\varphi(A) \neq e$, then $H_{1}(\overline{\nu A} ; \mathbb{Z}[G])=0$ since $\varphi$ is an element of infinite order since $G$ is torsion free. Furthermore $H_{1}(\partial(\overline{\nu A}))$ is a free $\mathbb{Z}[G]$-module on the meridian of $A$, which gets mapped to the longitude in $S^{3} \backslash \nu C$ which is zero in $H_{1}\left(S^{3} \backslash\right.$ $\nu C ; \mathbb{Z}[\mathbb{Z}])$. From the above commutative diagram it now follows that

$$
H_{1}\left(M_{S} ; \mathbb{Z}[G]\right) \cong H_{1}\left(M_{K} ; \mathbb{Z}[G]\right) \oplus H_{1}\left(S^{3} \backslash \nu C ; \mathbb{Z}[\mathbb{Z}]\right) \otimes_{\mathbb{Z}[\mathbb{Z}]} \mathbb{Z}[G]
$$

since $H_{1}\left(S^{3} \backslash \nu C ; \mathbb{Z}[G]\right) \cong H_{1}\left(S^{3} \backslash \nu C ; \mathbb{Z}[\mathbb{Z}]\right) \otimes_{\mathbb{Z}[\mathbb{Z}]} \mathbb{Z}[G]$. The lemma now follows from $H_{1}\left(S^{3} \backslash \nu C ; \mathbb{Z}[\mathbb{Z}]\right) \cong H_{1}\left(M_{C} ; \mathbb{Z}[\mathbb{Z}]\right)$.

\section{Examples: Satellite knots of $6_{1}$}

In Figure 7.1 we see three projections of the knot $6_{1}$, the first one having the minimal crossing number 6 . Indeed, the isotopy between $K_{1}$ and $K_{3}$ is shown in [2. The isotopy between $K_{2}$ and $K_{3}$ follows from Figures 1.1] and 7.2 , which shows that both knots are formed by band connected sum of two trivial knots 


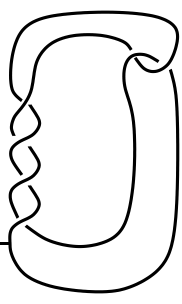

$K_{1}$

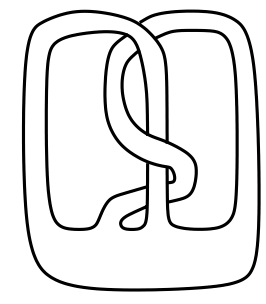

$K_{2}$

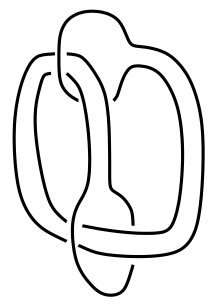

$K_{3}$

Figure 7.1: Three projections of the knot $6_{1}$

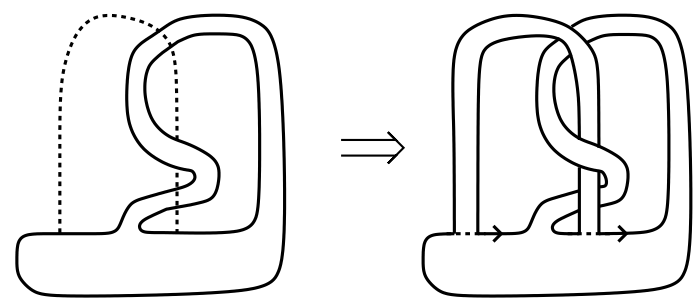

Figure 7.2: A ribbon disk for $6_{1}$

along isotopic bands. Note that the two trivial circles in Figure 7.2 bound disjoint disks in $S^{2}$. Now consider the knot $6_{1}$ with the Seifert surface $F$ in Figure 7.3. With the given basis $a, b$ for $H_{1}(F)$ we get

$$
A\left(6_{1}\right)=\left(\begin{array}{ll}
0 & 2 \\
1 & 0
\end{array}\right)
$$

which then shows that $\Delta_{61}(t)=\operatorname{det}\left(A t-A^{t}\right)=(t-2)\left(t^{-1}-2\right)$.

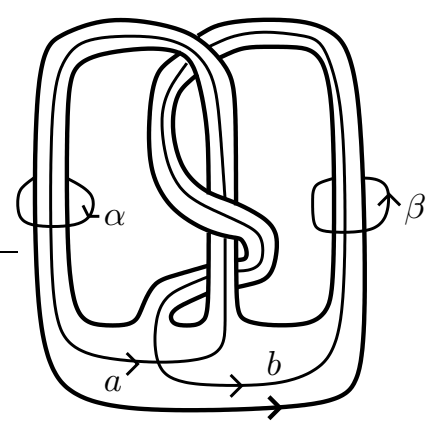

Figure 7.3: Seifert surface for $6_{1}$ 


\section{Examples of $\mathbf{h}-$ ribbon knots}

We first prove a general result. Let $R$ be a ribbon knot with ribbon disk $D_{R}$, such that $\pi_{1}\left(D^{4} \backslash D_{R}\right) \cong S R$. Denote the induced map $\pi_{1}\left(M_{K}\right) \rightarrow G:=S R$ by $\varphi$. Let $C$ be any knot and $A \subset S^{3} \backslash K$ the unknot in $S^{3}$.

Proposition 7.4 If $\varphi(A)=e$ then the satellite knot $S=S(R, C, A)$ is $h_{-}$ ribbon.

Proof By Lemma 6.2 there exists a map $\pi_{1}\left(M_{S}\right) \rightarrow G$ with $H_{*}\left(M_{S} ; \mathbb{Z}[G]\right) \cong$ $H_{*}\left(M_{R} ; \mathbb{Z}[G]\right)$. From Theorem 1.3 it follows that $\operatorname{Ext}_{\mathbb{Z}[G]}^{1}\left(H_{1}\left(M_{R} ; \mathbb{Z}[G]\right), \mathbb{Z}[G]\right)$ $=0$ and hence $\left(M_{S}, \varphi\right)$ also satisfies $\operatorname{Ext}_{\mathbb{Z}[G]}^{1}\left(H_{1}\left(M_{S} ; \mathbb{Z}[G]\right), \mathbb{Z}[G]\right)=0$. The proposition is implied by Theorem 1.3 .

This result is similar to the well-known fact that the Whitehead double of any knot (which is the satellite of the unknot) is topologically slice. This is an immediate corollary from Freedman's slicing theorem and Lemma 6.2.

Now turn back to the study of $K=6_{1}$. From the discussion in Section 2 we know that $K$ has a ribbon disk $D$ such that $\pi_{1}\left(N_{D}\right) \cong\left\langle a, b \mid a^{-1} b a^{-1} b a b^{-1}\right\rangle=$ $S R$. The group $\pi_{1}\left(M_{K}\right) \cong \pi_{1}\left(S^{3} \backslash K\right) /\langle$ longitude $\rangle$ is generated by the meridians of $K_{3}$. From Figure 2.3 it follows that the map

$$
\varphi: \pi_{1}\left(M_{K}\right) \rightarrow \pi_{1}\left(N_{D}\right) \cong\left\langle a, b \mid a^{-1} b a^{-1} b a b^{-1}\right\rangle=G
$$

is given by the map indicated in Figure 7.5. The image of the other meridians

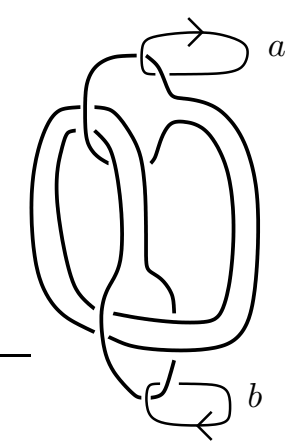

Figure 7.5: The map $\pi_{1}\left(S^{3} \backslash K\right) \rightarrow\left\langle a, b \mid a^{-1} b a^{-1} b a b^{-1}\right\rangle$

is determined by the image of the two given meridians.

As an example take $K=6_{1}, A$ as in Figure 7.6 and $C$ the trefoil knot. We 

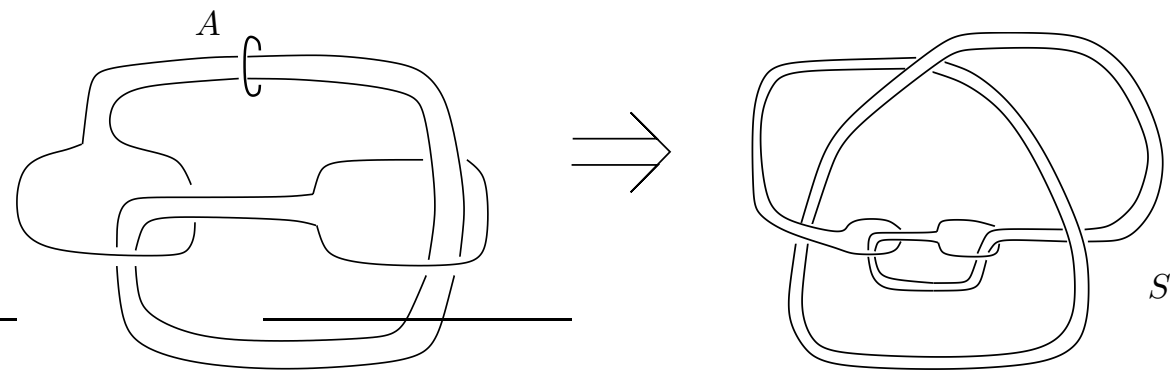

Figure 7.6: Ribbon knot with choice of $A$ and the satellite knot $S\left(6_{1}\right.$, trefoil, $\left.A\right)$

have $\varphi(A)=e \in G$ as one can easily verify. Therefore $S$ is $\mathrm{h}$-ribbon by Proposition 7.4. But in fact $S$ is trivially ribbon already, since the ribbon disk of $61_{1}$ 'survives' the satellite construction. We therefore need a more subtle choice of $A$. Now consider Figure 1.5. One can easily verify that $\varphi(A)=e$. The knot $6_{1}$ as a knot in the torus $S^{3} \backslash \nu A$ is given in Figure 1.5 on the right. Wrapping this knot around say the trefoil gives a h-ribbon knot, which we conjecture not to be smoothly ribbon.

Remark It follows immediately from arguments as in Lemma 6.2 that

$$
\left(H_{1}\left(M_{K} ; \mathbb{Z}[\mathbb{Z}]\right), B \ell(\mathbb{Z})\right) \cong\left(H_{1}\left(M_{S} ; \mathbb{Z}[\mathbb{Z}]\right), B \ell(\mathbb{Z})\right) .
$$

In particular $K$ and $S$ have the same abelian invariants. Livingston 21] showed that there exists a knot $\tilde{K}$ with

$$
\left(H_{1}\left(M_{K} ; \mathbb{Z}[\mathbb{Z}]\right), B \ell(\mathbb{Z})\right) \cong\left(H_{1}\left(M_{\tilde{K}} ; \mathbb{Z}[\mathbb{Z}]\right), B \ell(\mathbb{Z})\right)
$$

which is not topologically slice (cf also 18]). This shows that the vanishing of $B \ell(S R)$, which is a condition on the non-commutative homology of $K$, can not be reduced to a condition on abelian invariants.

\section{Non-existence of certain ribbon disks}

Now consider the knot $6_{1}$ with the Seifert surface $F$ in Figure 7.3. For given knots $C_{\alpha}, C_{\beta}$ consider $S\left(K, C_{\alpha}, C_{\beta}, \alpha, \beta\right)$, ie, the result of applying the satellite construction twice. This means that we tie knots $C_{\alpha}$ and $C_{\beta}$ into the bands $\alpha$ and $\beta$.

Proposition 7.7 If $\Delta_{C_{\alpha}}(t) \neq 1, \Delta_{C_{\beta}}(t) \neq 1$, then $S$ has no h-ribbon with fundamental group $S R$. 
Proof Assume that $S$ has in fact a h-ribbon $D$ with fundamental group $G:=$ $S R=\mathbb{Z} \ltimes \Lambda /(t-2)$. Then $\operatorname{Ker}\left\{H_{1}\left(M_{S} ; \mathbb{Z}[\mathbb{Z}]\right) \rightarrow H_{1}\left(N_{D} ; \mathbb{Z}[\mathbb{Z}]\right)\right\}$ is a metabolizer for $B \ell(\mathbb{Z})($ cf $[12])$. In the following we will write $\Lambda:=\mathbb{Z}\left[t, t^{-1}\right] \cong \mathbb{Z}[\mathbb{Z}]$. Note that $\alpha, \beta$ in Figure 7.3 lift to elements $\tilde{\alpha}, \tilde{\beta}$ in $H_{1}\left(M_{K} ; \Lambda\right)$, in fact

$$
H_{1}\left(M_{K} ; \Lambda\right) \cong(\Lambda \tilde{\alpha} \oplus \Lambda \tilde{\beta}) /\left(A t-A^{t}\right) \cong \Lambda \tilde{\alpha} /(2 t-1) \oplus \Lambda \tilde{\beta} /(t-2) .
$$

Furthermore the Blanchfield pairing $B \ell(\mathbb{Z})$ is given by the matrix $(t-1)(A t-$ $\left.A^{t}\right)^{-1}$. It is easy to see that the submodule generated by $\tilde{\alpha}$ respectively by $\tilde{\beta}$ are the only two metabolizers for $B \ell(\mathbb{Z})$. In particular the map $\pi:=\pi_{1}\left(M_{S}\right) \rightarrow$ $\pi_{1}\left(N_{D}\right)$ is up to automorphism of $G$ either of the form

$$
\varphi_{\alpha}: \pi_{1}\left(M_{S}\right) \rightarrow \pi / \pi^{(2)} \cong \mathbb{Z} \ltimes H_{1}\left(M_{S} ; \Lambda\right) \rightarrow \mathbb{Z} \ltimes \Lambda \tilde{\alpha} /(2 t-1) \rightarrow S R
$$

or it is of the same form with $\tilde{\alpha}$ replaced by $\tilde{\beta}$. We denote this homomorphism by $\varphi_{\beta}$. By Theorem 1.3 we get $\operatorname{Ext}_{\mathbb{Z}[G]}^{1}\left(H_{1}\left(M_{S} ; \mathbb{Z}[G]\right), \mathbb{Z}[G]\right)=0$ with $G-$ coefficients induced by $\varphi_{\alpha}$ or by $\varphi_{\beta}$. Now consider coefficients induced by $\varphi_{\alpha}$. From Lemma 6.2 it follows that

$$
H_{1}\left(M_{S} ; \mathbb{Z}[G]\right) \cong H_{1}\left(M_{K} ; \mathbb{Z}[G]\right) \oplus H_{1}\left(M_{C_{\alpha}} ; \mathbb{Z}[\mathbb{Z}]\right) \otimes_{\mathbb{Z}[\mathbb{Z}]} \mathbb{Z}[G] .
$$

We compute

$$
\begin{aligned}
& \operatorname{Ext}_{\mathbb{Z}[G]}^{1}\left(H_{1}\left(M_{S} ; \mathbb{Z}[G]\right), \mathbb{Z}[G]\right) \\
\cong & \left.\operatorname{Ext}_{\mathbb{Z}[G]}^{1}\left(H_{1}\left(M_{K} ; \mathbb{Z}[G]\right), \mathbb{Z}[G]\right) \oplus H_{1}\left(M_{C_{\alpha}} ; \mathbb{Z}[\mathbb{Z}]\right) \otimes_{\mathbb{Z}[\mathbb{Z}]} \mathbb{Z}[G], \mathbb{Z}[G]\right) \\
\cong & \operatorname{Ext}_{\mathbb{Z}[G]}^{1}\left(H_{1}\left(M_{K} ; \mathbb{Z}[G]\right), \mathbb{Z}[G]\right) \oplus \operatorname{Ext}_{\mathbb{Z}[G]}^{1}\left(H_{1}\left(M_{C_{\alpha}} ; \mathbb{Z}[\mathbb{Z}]\right) \otimes_{\mathbb{Z}[\mathbb{Z}]} \mathbb{Z}[G], \mathbb{Z}[G]\right) \\
\cong & \operatorname{Ext}_{\mathbb{Z}[G]}^{1}\left(H_{1}\left(M_{K} ; \mathbb{Z}[G]\right), \mathbb{Z}[G]\right) \oplus \operatorname{Ext}_{\mathbb{Z}[\mathbb{Z}]}^{1}\left(H_{1}\left(M_{C_{\alpha}} ; \mathbb{Z}[\mathbb{Z}]\right), \mathbb{Z}[\mathbb{Z}]\right) \\
\cong & \operatorname{Ext}_{\mathbb{Z}[\mathbb{Z}]}^{1}\left(H_{1}\left(M_{C_{\alpha}} ; \mathbb{Z}[\mathbb{Z}]\right), \mathbb{Z}[\mathbb{Z}]\right) .
\end{aligned}
$$

Note that $H_{1}\left(M_{C_{\alpha}} ; \mathbb{Z}[\mathbb{Z}]\right) \cong H_{1}\left(S^{3} \backslash C_{\alpha} ; \mathbb{Z}[\mathbb{Z}]\right)$, in particular it is $\mathbb{Z}$-torsion free. It follows from [20, Theorem 3.4] that $\operatorname{Ext}_{\mathbb{Z}[\mathbb{Z}]}^{1}\left(H_{1}\left(M_{C_{\alpha}} ; \mathbb{Z}[\mathbb{Z}]\right), \mathbb{Z}[\mathbb{Z}]\right) \cong$ $H_{1}\left(M_{C_{\alpha}} ; \mathbb{Z}[\mathbb{Z}]\right)$, which is non-trivial since $\Delta_{C_{\alpha}}(t) \neq 1$. The same calculation for $\varphi_{\beta}$ then gives the contradiction.

\section{Non-uniqueness of ribbon groups}

A ribbon knot can in general have ribbons with non-isomorphic groups. For example let $K$ be a non-trivial knot with $\Delta_{K}(t)=1$, eg, the Whitehead double of a non-trivial knot. Let $G:=\pi_{1}\left(S^{3} \backslash K\right)$, then $G$ is in particular a ribbon group for the knot $L:=K \#-K$. On the other hand, $L$ still has trivial Alexander polynomial and so by Theorem 1.3 it follows that $L$ also has a $\mathrm{h}^{-}$ ribbon with fundamental group $\mathbb{Z}$. In fact, if $K$ is the Whitehead double of a ribbon knot then it is ribbon, not just h-ribbon, with fundamental group $\mathbb{Z}$ (and so is $L$ ). 


\section{References}

[1] C S Aravinda, F T Farrell, S K Roushon, Surgery groups of knot and link complements, Bull. London Math. Soc. 29 (1997) 400-406 MathReview

[2] A J Casson, C M Gordon, Cobordism of classical knots, from: "À la recherche de la topologie perdue", Progr. Math. 62, Birkhäuser Boston, Boston, MA (1986) 181-199 MathReview

[3] T D Cochran, Noncommutative knot theory, Algebr. Geom. Topol. 4 (2004) 347-398 MathReview

[4] T D Cochran, K E Orr, P Teichner, Structure in the classical knot concordance group, Comment. Math. Helv. 79 (2004) 105-123 MathReview

[5] T D Cochran, K E Orr, P Teichner, Knot concordance, Whitney towers and $L^{2}$-signatures, Ann. of Math. (2) 157 (2003) 433-519 MathReview

[6] T D Cochran, $\mathbf{P}$ Teichner, Knot concordance and von Neumann $\rho$-invariants, preprint (2004)

[7] T D Cochran, T Kim, Higher-order Alexander invariants and filtrations of the knot concordance group, preprint (2005)

[8] J Duval, Forme de Blanchfield et cobordisme d'entrelacs bords, Comment. Math. Helv. 61 (1986) 617-635 MathReview

[9] MH Freedman, The topology of four-dimensional manifolds, J. Differential Geom. 17 (1982) 357-453 MathReview

[10] M H Freedman, F Quinn, Topology of 4-manifolds, Princeton Mathematical Series 39, Princeton University Press, Princeton, NJ (1990) MathReview

[11] M H Freedman, P Teichner, 4-manifold topology. I. Subexponential groups, Invent. Math. 122 (1995) 509-529 MathReview

[12] S Friedl, Eta invariants as sliceness obstructions and their relation to CassonGordon invariants, Algebr. Geom. Topol. 4 (2004) 893-934 MathReview

[13] S Garoufalidis, P Teichner, On knots with trivial Alexander polynomial, J. Differential Geom. 67 (2004) 167-193 MathReview

[14] RE Gompf, Smooth concordance of topologically slice knots, Topology 25 (1986) 353-373 MathReview

[15] R E Gompf, A I Stipsicz, 4-manifolds and Kirby calculus, Graduate Studies in Mathematics 20, Amer. Math Soc. Providence, RI (1999) MathReview

[16] J Howie, On locally indicable groups, Math. Z. 180 (1982) 445-461 MathReview

[17] J Howie, On the asphericity of ribbon disc complements, Trans. Amer. Math. Soc. 289 (1985) 281-302 MathReview

[18] T Kim, An infinite family of non-concordant knots having the same Seifert form, Comment. Math. Helv. 80 (2005) 147-155 MathReview 
[19] J Levine, A method for generating link polynomials, Amer. J. Math. 89 (1967) 69-84 MathReview

[20] J Levine, Knot modules. I, Trans. Amer. Math. Soc. 229 (1977) 1-50 MathReview

[21] C Livingston, Seifert forms and concordance, Geom. Topol. 6 (2002) 403-408 MathReview

[22] C Livingston, Computations of the Ozsváth-Szabó knot concordance invariant, Geom. Topol. 8 (2004) 735-742 MathReview

[23] P Ozsváth, Z Szabó, Knot Floer homology and the four-ball genus, Geom. Topol. 7 (2003) 615-639 MathReview

[24] A A Ranicki, Algebraic L-theory. III. Twisted Laurent extensions, from: "Algebraic K-theory, III", SLN 343, Springer (1973) 412-463 MathReview

[25] A A Ranicki, Algebraic L-theory and topological manifolds, Cambridge Tracts in Mathematics 102, Cambridge University Press (1992) MathReview

[26] A Ranicki, High-dimensional knot theory, Springer Monographs in Mathematics, Springer-Verlag, New York (1998) MathReview

[27] A Ranicki, Algebraic and geometric surgery, Oxford Mathematical Monographs, Oxford University Press (2002) MathReview

[28] J A Rasmussen, Khovanov homology and the slice genus, e-print (2004) arXiv:math.GT/0402131

[29] S K Roushon, Topology of 3-manifolds and a class of groups II, preprint (2003)

[30] R Strebel, Homological methods applied to the derived series of groups, Comment. Math. Helv. 49 (1974) 302-332 MathReview

[31] C T C Wall, Surgery on compact manifolds, Mathematical Surveys and Monographs 69, Amer. Math. Soc. Providence, RI (1999) MathReview

[32] J S Wilson, Soluble groups of deficiency 1, Bull. London Math. Soc. 28 (1996) 476-480 MathReview 